\title{
Person-Centered-Care Climate in a Tertiary Hospital: Staff Perspective
}

\author{
Khaled Al-Surimi $\mathbb{D}^{1-3}$ \\ Zahra Alhayek ${ }^{1,2}$ \\ David Edvardsson ${ }^{4}$ \\ Badr Al-Khateeb ${ }^{1,2}$ \\ Naila Shaheen $\mathbb{D}^{1,5}$
}

'King Saud bin Abdulaziz University for Health Sciences, Riyadh, Kingdom of Saudi Arabia; ${ }^{2}$ King Abdullah International Medical Research Center, Riyadh, Kingdom of Saudi Arabia; ${ }^{3}$ Faculty of Medicine and Health Sciences, Thamar University, Dhamar, Yemen; ${ }^{4}$ Department of Nursing, Umea University, Umea, Sweden; ${ }^{5}$ Department of Biostatistics and Bioinformatics, King Abdullah International Medical Research Center, Riyadh, Kingdom of Saudi Arabia
Correspondence: Naila Shaheen Email drnaila@hotmail.com
Introduction: Most health care systems strive to improve the quality, safety and value of healthcare, with an emphasis on moving towards patient-centered care/person-centered care (PCC) approach. The aim of the current study was to assess health care providers' perspectives on PCC climate in hospital setting and to identify the role of providers in determining the perception of the PCC climate.

Methods: A survey, using person-centered climate questionnaire-staff version, was employed in health care providers of a tertiary care hospital. Data included variables age, gender, education level, occupation, and years of experience and three PCC dimensions. PCC overall and subscale scores were reported as mean and standard deviation. Factors associated with PCC climate perception were analyzed using a Poisson model.

Results: Out of 1216 respondents; the majority $47 \%$ aged between 18 and 34 years; $79 \%$ women, $68 \%$ were nurses. The overall mean score was $45.96 \pm 15.36$ (range $0-70$ ). Subscale scores were Safety $20.15 \pm 5.0$ (range 0-30), Everydayness $12.02 \pm 3.52$ (range 0-20) and Community 13.79 \pm 3.34 (range 0-20). Increasing age was a significant factor associated with PCC scores for the overall, safety, everydayness, and community scales, with a positive association. Lower scores were reported more by women compared with men, for overall $(p=0.0005)$, and everydayness $(p=0.006)$ scales. Higher safety scores were reported by health care providers with a diploma compared to master's degree $(p=0.009)$, Ph.D. $(p=0.007)$, for technicians compared with nurses $(p=0.007)$, and for day shift compared with day/night shift workers $(\mathrm{p}=0.025)$. PCC scores were not significantly different across health care providers' years of experience.

Conclusion: There is a room for PCC climate improvement based on the low scores compared to the literature. The study findings indicated that the main factors associated with HCPs' perception of PCC were higher age and female gender, and these factors would benefit from further research.

Keywords: health care providers' perception, person-centered care, psychosocial climate, tertiary care facility, work climate

\section{Plain Language Summary}

This is a first study focusing on the healthcare workers thinking of the person-centered care climate in a hospital in Saudi Arabia. The person centered is a new approach which focuses on making the person and patient the center of healthcare decision making. The authors have assessed the personcentered care climate using a well-known validated tool measuring three dimensions: Safety, Everydayness, and Community. Each one of these dimensions has been measured by the number of items. The authors have found that the reported scores were low in all dimensions of the personcentered care. The reported scores were higher with an increase in age. Female health care workers had reported low scores on environmental factors. The results can provide empirical evidence for 
initiating health policy and practices to improve the person-centered care along with the quality of provided care in the hospital and the health outcomes.

\section{Introduction}

Most health care systems strive to improve the quality and safety of the healthcare they provide, ${ }^{1}$ with more emphasis on moving toward patient-centered care/person-centered care(PCC) approach as a way to improve the quality, safety and patient outcomes for both patients and Health Care Providers (HCPs). ${ }^{2}$ PCC has been defined by the Institute of Medicine (IOM) as providing care that is respectful of, and responsive to, individual patient preferences, needs and values, and ensuring that patient values guide all clinical decisions. ${ }^{3}$

In 2004, IOM published a report exploring patient safety and asserted that the work environment culture is a substantial factor affecting the quality and patient safety of health care delivery. ${ }^{4}$ PCC have been described to improve HCPs' job satisfaction, ${ }^{5}$ reduce negligence complaints, ${ }^{6}$ reduces health care $\operatorname{cost}^{7}$ and job satisfaction and turnover intention among staff. ${ }^{8,9}$ There are many definitions and views published in the literature on PCC. ${ }^{9}$ There is a growing interest in incorporating the PCC approach into healthcare policymaking and daily practice; however, a clear definition and specification of its elements remain uncertain which resulted in inconsistency when implementing PCC. ${ }^{10}$ As per the World Health Organization (WHO), 2015 report the PCC is defined as

Care approaches and practices that see the person as a whole with many levels of needs and goals, with these needs coming from their own personal social determinants of health. ${ }^{11}$

A literature review has summarized the available definitions for PCC, with a key message that PCC also builds on a relationship between patient as a person and a caregiver.${ }^{12}$ It has been reported that patient-centered care or personcentered care (PCC) is used interchangeably throughout the literature, and some researchers have suggested that the definition is the same, ${ }^{13-15}$ with a lack of agreement across published articles. ${ }^{15,16}$ Some researchers have pointed out that the term person-centered shifts attention from the illness to the individual; with a greater focus on the person who is experiencing the illness. ${ }^{14}$

This paper recognizes that even if there may be substantial overlap between the descriptors of patient and person-centered care concepts, the concept of person is more aligned with the underlying humanistic PCC philosophy of building care on the values, preferences, needs and priorities of the person in need of care and support as proposed by WHO, and as such, the concept of personcentered care will be used throughout this paper.

It is worth mentioning that the PCC approach has been introduced as a quality indicator, where health care is tailored to the individual's needs and being responsive to preferences, and hence PCC is seen as a cooperation and collaboration between HCPs and the patient as a person in need of care. ${ }^{9}$ Kitson et al have identified three elements of PCC: the patient as a person's involvement in his/her care, the interaction between the patient as a person and HCP, and the context and work environment, where healthcare is happening. ${ }^{17}$ The work environment has been described as influencing not only staff satisfaction and turn-over but also the quality of care and patient safety. The work climate and culture of the organization are commonly used to describe staff perception of their work setting. ${ }^{17}$ Organizational culture has been described as more focused on shared values, assumptions, beliefs, myths, and traditions, which when combined can lead the employee to behave in a certain manner. On the other hand, organizational climate has been defined as a widespread perception of the employees about their work environment. Numerous factors have been shown to determine an organization's climate: leadership, organizational structure, historical forces, the standard of accountability, behavior, and communication, and these factors have had an impact on the organization's climate. ${ }^{18}$

Several studies have been conducted to specify and delineate which factors have influenced the implementation of PCC. ${ }^{9}$ These studies report that work environment and organizational factors had stronger influences on PCC, ${ }^{19}$ in which the organizational system fosters shared decision making and facilitates the PCC process through activities. Additionally, the concept and practice of PCC have been described as essential for being incorporated into all organizational policies and procedures. ${ }^{20,21}$ However, the focus of PCC at the organizational level to improve quality and health outcomes has been lacking, and there still remains unknown knowledge that quality of care is affected by the organizational environment or culture. $^{22}$

Research concerning evidence of the link between organizational culture and quality and patient safety ${ }^{23}$ still remain limited, although some studies showed positive impact of improving PCC climate on patient and staff 
satisfaction and outcomes is clearly evident in the literature. $^{8}$

It is not indisputable that the person in need of care is the most important and reliable measure for measuring PCC in health care organizations. However, measuring the perspective of HCPs on the person-centered work climate is also crucial. ${ }^{13}$ Studies have measured patient experience in terms of PCC, but there is a gap in the literature regarding the HCPs' perception. ${ }^{2}$ Berghout et al have reported person-centered care as key elements identified by healthcare professionals as treating patients with dignity and respect, an interdisciplinary approach and as having equal access and good outcomes from care. However, the study was limited to geriatric departments only. $^{26}$ The literature is scarce on reporting HCPs' perception of PCC, which suggests a need to measure HCPs' perception of their work climate that supports and facilitates policies and practices of PCC approach. Research evidence showed that the provision of both PCC and individualized care is associated with the care environment climate. $^{24,25}$ The aim of the current study was to assess health care providers' perspectives on person-centered care climate and to identify the role of health care providers' characteristics in determining the perception of a person-centered care climate in the study hospital.

\section{Materials and Methods Study Design and Participants}

This was a cross-sectional survey of HCPs' perceptions conducted in a tertiary care hospital setting. This tertiary care hospital employs more than 7000 different health care providers, including doctors, nurses, therapists, technicians, and others, with a total bed capacity exceeding 1000 beds. The hospital is accredited by the Joint Commission International.

A list of all HCPs working in the tertiary care hospital was obtained from the employee relation office. The sampling frame was derived from the list of HCPs' email IDs. The study enrolled male and female HCPs, all of whom were full-time staff who had completed three months of employment and who were directly involved in patient care. The administrative staff was excluded. An email was sent to all the HCPs with the study introduction, including the consent form. The HCPs were requested to complete an electronic survey following their agreement to participate in the study. The electronic survey was used for data collection. The responses with missing data were excluded. Ethical approval was obtained from the King Abdullah International Medical Research Center Institutional Review Board with approval number (SP16/ 078). This study was conducted in accordance with the Declaration of Helsinki. All participants were adequately informed of the aims, methods, and risks of the study as well as of voluntary participation and confidentiality of the responses at the introduction of the survey. The responses were anonymous and participants' confidentiality was maintained.

\section{Sample Size, Sampling Technique}

The adequate minimum sample size was calculated, assuming alpha level 0.05 , 95\% confidence interval, a margin of error $5 \%$, the estimated sample size was 385. The sample size was adjusted for $30 \%$ non-response rate to the required sample size as 550 subjects. Data was collected via online survey in English language. The survey consisted of two parts. The first part included questions about demographic variables age, gender, nationality, occupation, position, work experience, working unit, working shifts, and interaction with patients. The second part is about the PCC environment, which was measured by a questionnaire called Person Centered Climate Questionnaire-staff version (PCCQ-SV).

\section{Instrument}

The Person-Centered Climate Questionnaire-staff version (PCCQ-SV) as developed by Edvardsson et $\mathrm{al}^{21}$ was used in the study. This tool aims primarily to measure the Person-Centered Climate from a staff perspective, and consists of three main scales, namely Safety, Everydayness, and Community. The previous studies that used the PCCQ-SV found that PCCQ-SV has good reliability (Cronbach's $\alpha=0.88$ ). Overall, the PCCQ consists of 14 items measure the overall PCC climate. The total score ranging from 14 to 84 where the higher score corresponds to a higher person-centered climate. Edvardsson et al reported a cut-off score of $\leq 49$ is considered "well below average", score 50-56 "below average", 57-62 "above average" and $\geq 63$ "well above average". 17

A validated tool, the Person-Centered Climate Questionnaire-Staff version (PCQ-S) and a set of questions composed on demographics (items $=5$ ), and employment area/employment experience (items $=6$ ) was used as the study survey. The PCQ-S is a reliable and valid tool (Cronbach's $\alpha=0.88)^{17,21}$ and consists of the three subscales; safety (items $=1-6)$, everydayness (items $=7-10)$, 
and community (items $=11-14$ ). The items were scored on a Likert scale, the values were ordinal, ranging from 0 (no, I disagree completely) to 5 (yes, I agree completely). The overall PCC climate is measured by all 14 items, with a score ranging from zero to 70 where the higher score denotes a more person-centered climate. ${ }^{17}$ The data were reviewed for accuracy and validity prior to statistical analysis. Surveys with incomplete responses were excluded. A pilot testing conducted before embarking on this main study showed that the study instrument was valid and reliable, having good internal consistency to be used in our study context. The overall reliability scale was 0.90 , and for the subscales, as follows: Safety (0.82), Everydayness (0.82), and Community (0.79).

\section{Statistical Analysis}

The categorical variables gender, age, nationality, marital status, occupation, level of education, work experience, working shift, and working unit were reported in frequency tables and as percentages. PCQ-S subscale scores were summarized and reported as mean, standard deviation (SD), and range. HCPs' views on person-centered care climate dimensions were summarized as agree ("No I partly agree", "Yes I agree" and "Yes I agree completely") and disagree ("No I disagree completely", "No I disagree", "No I partly disagree").

Since the data had followed poisson distribution, poisson regression was used to identify the independent factors associated with the PCC perception for overall, safety, everydayness, and community subscales. The independent variables (age, gender, education, occupation, working shift, and years of experience in the institute) were selected after running the univariate analysis. The significant variables on the univariate analysis were finalized. Whether a participant had an interaction with patients or no was not significant for all the subscale scores so was not included in the model. The independent variables were assessed for collinearity and interaction. Results were reported as ratios, corresponding 95\% CI and p-values. P-value less than 0.05 was considered significant. Statistical analyses were carried out using SAS version 9.4 (SAS Institute, Cary, NC, USA).

\section{Results}

\section{Participants' Characteristics}

A total of 1430 respondents responded to the email, and 1359 (95\%) agreed to participate in the study. A number of $143 / 1359(10 \%)$ surveys with incomplete responses were excluded, resulting in a study sample of 1216 participants. The response rate was 95\% (1359/1430). The participants' characteristics are presented in Table 1. Most of the participants were women $79 \%$. In terms of age, the largest group of participants' $47 \%$ were aged between 18 and 34 years. Moreover, more than two-thirds of participants $80 \%$ were non-Saudis. More than half $654 \%$ were married. $74 \%$ had a bachelor's degree. The majority of the participants $52 \%$ were working in in-patient units. Almost $60 \%$ of respondents reported working day and night shifts. The majority of the participants $90 \%$ stated that they had interaction with patients.

\section{Degree of Person-Centeredness Climate}

The average overall person-centered climate score reported by HCPs was $45.96 \pm 10.15$ (range 10-70). The scores for individual items on the safety scale varied from 3.28 to 3.70 ; combining to give a mean score for the safety scale of $20.15 \pm 5.0$ (range $0-30$ ). The individual scores on the everydayness scale varied from 2.63-3.58; and the mean score for the scale was $12.02 \pm 3.52$ (range 0-20). The individual scores on the community scale varied from 3.37 to 3.5 , and the mean community scale score was $13.79 \pm 3.34$ (range 1-20) (Table 2).

\section{Health Care Providers' Perception of Person-Centered Care Climate \\ Safety Scale}

With regard to the safety scale, $90 \%$ agreed that they feel welcome in their institution, $87 \%$ agreed that their institution acknowledge them as a person, $77 \%$ agreed that they felt to be themselves, $90 \%$ agreed that the patients were in safe hands in their institution, and lastly, $80 \%$ agreed that the language used by the staff of the institution was easily understood by the patients. The responses were good for all items on the safety scale (Table 3 ).

\section{Everydayness Scale}

In response to everydayness scale, $69 \%$ agreed that the institution felt homely, $75 \%$ agreed that there was something nice to look at in the institution, $65 \%$ agreed that the workplace was quiet and peaceful, $61 \%$ agreed that in the workplace they could forget about unpleasant thoughts, and lastly, $89 \%$ agreed that the institution was clean and neat. The responses were good for something nice to look at and neatness (Table 3). 
Table I Demographic Characteristics of the Study Participants

\begin{tabular}{|c|c|c|}
\hline \multicolumn{2}{|l|}{ Demographic Characteristics } & \multirow{2}{*}{$\begin{array}{c}\mathbf{n}=1216 \\
572(47.04) \\
397(32.65) \\
179(14.72) \\
68(5.59)\end{array}$} \\
\hline Age $n(\%)$ & $\begin{array}{l}18-34 \text { years } \\
35-44 \text { years } \\
45-54 \text { years } \\
\geq 55 \text { years }\end{array}$ & \\
\hline Gender $n(\%)$ & $\begin{array}{l}\text { Female } \\
\text { Male }\end{array}$ & $\begin{array}{l}958(78.78) \\
258(21.22)\end{array}$ \\
\hline Nationality $n(\%)$ & $\begin{array}{l}\text { Saudi } \\
\text { Non-Saudi }\end{array}$ & $\begin{array}{l}24 I(19.82) \\
975(80.18)\end{array}$ \\
\hline Marital status $\mathrm{n}(\%)$ & $\begin{array}{l}\text { Married } \\
\text { Single } \\
\text { Divorced/widower }\end{array}$ & $\begin{array}{c}66 I(54.36) \\
489(40.21) \\
66(5.43)\end{array}$ \\
\hline Level of education $n(\%)$ & $\begin{array}{l}\text { High school } \\
\text { Diploma } \\
\text { Bachelor degree } \\
\text { Master's degree } \\
\text { PhD or equivalent }\end{array}$ & $\begin{array}{c}20(1.65) \\
111(9.14) \\
907(74.65) \\
108(8.89) \\
69(9.14)\end{array}$ \\
\hline Occupation n(\%) & $\begin{array}{l}\text { Nurses/Nurse assistant } \\
\text { Technician* } \\
\text { Physician } \\
\text { Paramedic*** } \\
\text { Pharmacist }\end{array}$ & $\begin{array}{l}830(68.26) \\
125(10.28) \\
113(9.29) \\
97(7.89) \\
51(4.19)\end{array}$ \\
\hline Interaction with patients $\mathrm{n}(\%)$ & $\begin{array}{l}\text { Yes } \\
\text { No }\end{array}$ & $\begin{array}{c}1094(89.97) \\
122(10.03)\end{array}$ \\
\hline Working shift $\mathrm{n}(\%)$ & $\begin{array}{l}\text { Day and night shifts } \\
\text { Day shifts } \\
\text { Night shifts }\end{array}$ & $\begin{array}{c}753(61.92) \\
444(36.51) \\
19(1.56)\end{array}$ \\
\hline Working unit $n(\%)$ & $\begin{array}{l}\text { Inpatient } \\
\text { Others } \\
\text { Outpatient } \\
\text { Emergency room } \\
\text { Laboratory department } \\
\text { Pharmacy } \\
\text { Imaging department }\end{array}$ & $\begin{array}{c}640(52.63) \\
188(15.46) \\
178(14.64) \\
95(7.81) \\
44(3.62) \\
37(3.04) \\
34(2.80)\end{array}$ \\
\hline Work experience $n(\%)$ & $\begin{array}{l}\text { Less than } 5 \text { years } \\
6-10 \text { years } \\
\text { More than } 10 \text { years }\end{array}$ & $\begin{array}{l}218(17.93) \\
425(34.95) \\
573(47.12)\end{array}$ \\
\hline Working years in the current institution $n(\%)$ & $\begin{array}{l}\text { Less than I year } \\
1-3 \text { years } \\
4-6 \text { years } \\
7-10 \text { years } \\
\text { More than } 10 \text { years }\end{array}$ & $\begin{array}{c}73(6.0) \\
394(32.40) \\
303(24.92) \\
221(18.17) \\
225(18.50)\end{array}$ \\
\hline
\end{tabular}

Notes: *Technicians (pharmacy/laboratory/ patient care technicians). ***Paramedics (paramedics/midwives/physiotherapists/perfusionists/dentists/phlebotomists).

\section{Community Scale}

Regarding community scale, $84 \%$ agreed that in the workplace patients were able to stay in contact with their loved ones, $84 \%$ agreed that it was easy for patients to receive visitors in their institution, $86 \%$ agreed that it was easy for patients to talk with staff in their institution, and lastly, 
Table 2 Person-Centered Climate Scores

\begin{tabular}{|l|c|c|c|c|}
\hline Scores & Overall & Safety Scale & Everydayness Scale & Community Scale \\
\hline Median(IQR) & $46.0(13)$ & $21.0(7)$ & $12.0(4)$ & $14.0(4)$ \\
Mean \pm SD & $45.96 \pm 10.15$ & $20.15 \pm 5.0$ & $12.02 \pm 3.52$ & $13.79 \pm 3.34$ \\
Range & $10-70$ & $0-30$ & $0-20$ & $1-20$ \\
\hline
\end{tabular}

Table 3 Health Care Providers' Views on Person-Centered Care Climate Dimensions

\begin{tabular}{|c|c|c|c|c|}
\hline \multicolumn{2}{|c|}{ Person-Centered Care Climate Dimensions/Items } & \multirow{2}{*}{$\begin{array}{c}\text { n(\%) } \\
\text { Agree } \\
1101(90.54)\end{array}$} & \multirow{2}{*}{$\begin{array}{c}\text { n(\%) } \\
\text { Disagree }\end{array}$} & \multirow{2}{*}{$\begin{array}{c}\begin{array}{c}\text { Mean } \\
( \pm S D)\end{array} \\
3.63(0.94)\end{array}$} \\
\hline Safety & A place where I feel welcome & & & \\
\hline & A place where I feel acknowledged as a person & 1059(87.09) & $157(|2.9|)$ & $3.46(1.04)$ \\
\hline & A place where I feel I can be myself & $933(76.73)$ & $283(23.27)$ & $3.19(1.20)$ \\
\hline & A place where the patients are in safe hands & $1096(90.21)$ & $119(9.79)$ & $3.70(0.98)$ \\
\hline & A place where the staff use a language that the patients can understand & $997(80.35)$ & $239(19.65)$ & $3.28(1.16)$ \\
\hline \multirow[t]{5}{*}{ Everydayness } & A place which feels homely even though it is in an institution & $843(69.33)$ & $373(30.67)$ & $2.89(1.21)$ \\
\hline & A place where there is something nice to look at & $919(75.58)$ & $297(24.42)$ & $3.06(1.15)$ \\
\hline & A place where it is quiet and peaceful & $789(64.94)$ & $426(35.06)$ & $2.76(1.23)$ \\
\hline & A place where it is possible to get unpleasant thoughts out of your head & $739(60.77)$ & $477(39.23)$ & $2.63(1.18)$ \\
\hline & A place which is neat and clean & 1087(89.39) & $129(\mid 0.61)$ & $3.58(0.99)$ \\
\hline \multirow[t]{4}{*}{ Community } & A place where it is easy for the patients to keep in contact with their loved ones & 1027(84.46) & $189(15.54)$ & $3.40(1.04)$ \\
\hline & A place where it is easy for the patients to receive visitors & $1020(83.88)$ & $196(16.12)$ & $3.48(1.11)$ \\
\hline & A place where it is easy for the patients to talk to the staff & 1047(86.10) & $169(13.90)$ & $3.54(1.06)$ \\
\hline & A place where the patients have someone to talk to if they so wish & $1009(82.98)$ & $207(17.02)$ & $3.37(1.06)$ \\
\hline
\end{tabular}

$83 \%$ agreed that patients had someone to talk with in the institution if they so wished. The responses were good for all items on the community scale (Table 3 ).

\section{Factors Associated with Health Care Providers Perception of Overall PCC Scores}

No collinearity was observed between the independent variables. The poisson model had shown $\left(\mathrm{R}^{2}=0.06\right)$. The AIC for overall score was $(\mathrm{AIC}=9489.4079)$, safety scale $(\mathrm{AIC}=7354.0201)$, Everydayness $(\mathrm{AIC}=6486.0799)$, and community scale $(\mathrm{AIC}=6371.5791)$.

As summarized in Table 4, age was a significant factor when all age categories were compared with the age category 55 years and above, with higher PCC scores for overall, safety, everydayness, and community scales in older age group. Lower PCC scores were reported by women compared with men, for overall scale Rate Ratio (RR) 0.95 , 95\% CI (0.94-0.98), and everydayness scale RR (0.93), 95\% CI (0.9-0.98). However, no significant difference in PCC scores was observed between women and men on the safety and community scale. A statistically significant difference was observed for the overall scale scores of HCPs with the level of education; master's degree vs diploma RR (0.94), 95\% CI (0.91-0.99), and Ph.D. vs diploma RR 0.9, 95\% CI (0.87-0.98). Technicians reported higher PCC scores compared to nurses RR $(1.06$, 95\% CI (1.02-1.11)) on the safety scale. Timings of working shift impacted having more overall scale score for day shift RR (0.97), 95\% CI (0.96-1), as well as safety scale score RR (0.96), 95\% CI (0.94-1). The PCC scores were not significantly different across HCPs' years of experience at the institute.

\section{Discussion}

The aim of the current study was to assess health care providers' perspectives on person-centered care and to identify if health care providers' characteristics were related to the perception of a person-centered care climate. It was shown that the perception of health care providers for person-centered care climate was comparatively low across all scales compared with those reported in the literature. In terms of characteristics associated with person-centered care, it was found that a higher age was a significant predictor for higher scores on all scales. 


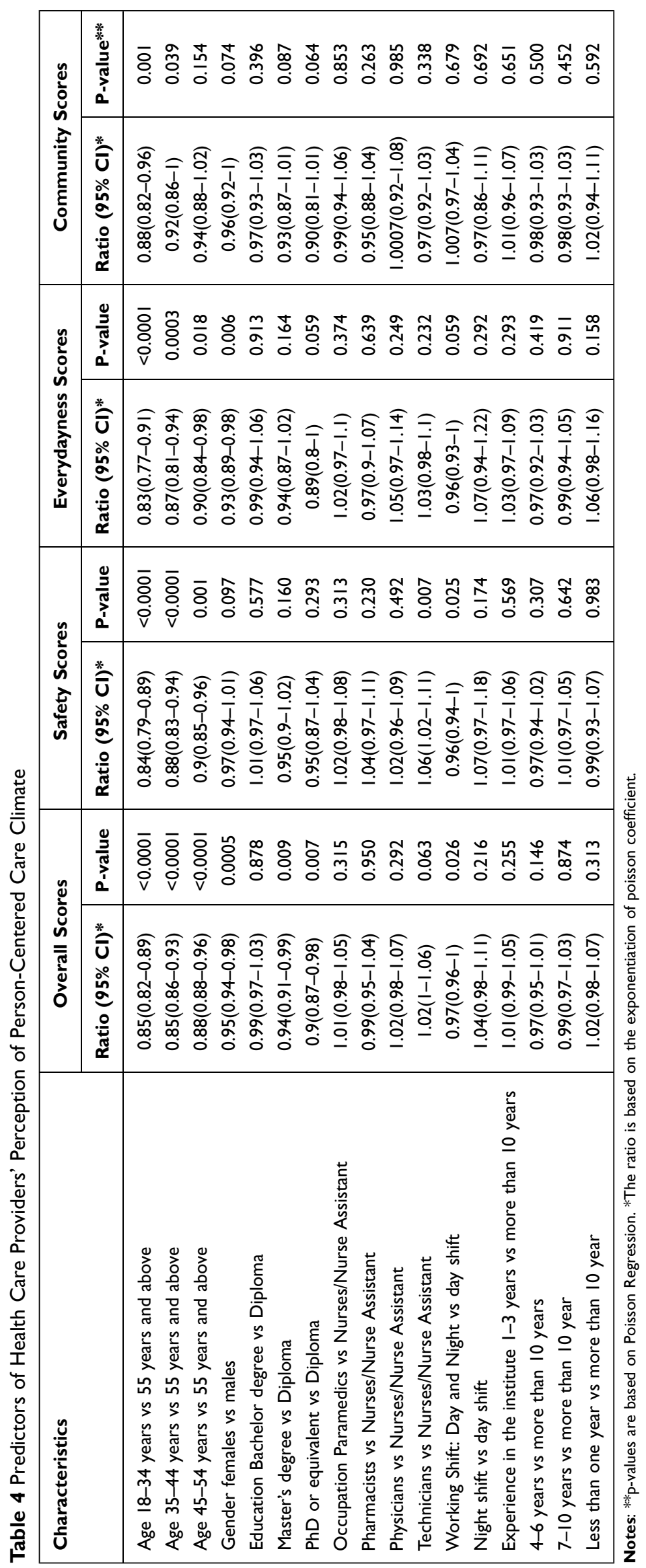


Females reported lower scores on the overall and everydayness scale. Higher safety scores were reported by technicians, staff with diplomas and day shift workers on the safety scale. The study fills a gap in the literature of HCPs' perception of person-centered care across different health care disciplines, cultures and contexts.

Several previous studies have assessed PCC in various populations, for example, a systematic review identifying 3045 articles reporting patients' perception of PCC. ${ }^{6}$ However, the perception of PCC from the perspectives of HCPs has not been widely assessed in the published literature, hence indicating a need for such studies. As PCC is a multidimensional concept build on humanistic theory of care and experiences of illness; this suggests an essential role for HCPs in understanding and promoting PCC. Berghout et al is one of the few researchers who have explored the importance of PCC from HCPs' perspectives, and identified treating patients with dignity and respect, having an interdisciplinary approach, and providing equal access and good outcomes from care as key elements of PCC. ${ }^{26}$ Furthermore, perceptions of PCC by health care staff was also assessed in a study conducted by Edvardsson et al in residential care units for the elderly. The mean scores for the overall scale and three subscales were higher than those in our study (overall; 55 vs 46, safety; 22 vs 20, everydayness; 17 vs 12 , and community; 18 vs 13). ${ }^{17}$ Potentially this can be related to the long-term residential nature of the context in which that study was conducted.

It has been stated that PCC is a fundamental aspect of the quality of care provided to people in need of care. ${ }^{26}$ As HCPs' have a constitutive role in delivering PCC, their perception is arguably fundamental for improvement of the quality of care provided to patients. ${ }^{26}$ The patient as a person is considered an active participant in care in PCC theory. ${ }^{27}$ In this study the HCPs' mostly agreed to the items in all three scales of the person-centered climate questionnaire-staff version.

The safety scale measures the perception of organizational safety. With regard to their perception of the safety scale, $90 \%$ of HCPs agreed that their institution was "a place where I feel welcome", and that "if they feel that patients are in safe hands". Those findings may indicate that the organization's climate is indeed perceived as safe and welcoming to staff as well as to patients. A study by Jorm et al tried to answer the question of whether patient safety should be more patient-centered, and suggested improvements based on a patient-centered safety model that also includes the patient in care activities. The author also highlighted that the patient safety model is incomplete if it does not address the patient's experiences. ${ }^{28}$ Thus, even if the concept of patient-centered care was used in that study, it raises the need for precise measurements of the relationship between safety culture, its impact on patient care, and the role of patient-centered or person-centered care, if assuming that these concepts are largely overlapping.

The second scale, everydayness, measures the perception by HCPs' of their working unit as homely, nicely decorated, with a calming atmosphere, and neat/clean. The HCPs' perception was good for "a place where there is something to nice look at it", and "a place which is neat and clean". Harris et al highlighted that the social environment is a major factor in PCC. Other studies have also shown that social perceptions of support plays a crucial role in patients' experiences of wellbeing, both physically and psychologically. ${ }^{29}$ This indicates the importance of assessing and monitoring the social experiences of the patient in relation to the environment and significant others; staff can enable patients to keep in contact with their families during hospitalization to facilitate experiences of everydayness. This was partly also highlighted in the third scale concerning the community climate, with additional statements measuring the extent to which HCPs perceive that it is easy for patients to keep in contact with their families, receive visitors, and talk with staff as well as others. The community climate low scores indicate that patients in contact with the family need improvement. In addition, the relationship between staff characteristics and perception of PCC across HCPs was also examined in this study. The main factors associated with HCPs' perception of PCC were age, and gender. The level of education and shift times were not associated with significant differences in scale scores except overall scores. Technicians did report different scores on the safety scale compared with nurses, and HCPs' age was identified as significant factors associated with their perception of PCC across all scales. Female participants had lower scores across overall and everydayness scales compared to males. However, a study by Sjogren et al had reported no significant impact of age on PCC scores, but females had scored more favorably compared to males. Moreover, Sjogren et al had assessed some additional covariates: job strain, work satisfaction, and psychosocial climate. $^{20}$

The literature is not rich with reports on perceived PCC among HCPs. Some of the available studies have described different methodological approaches ${ }^{26}$ or comparing PCC for specific groups and setting. ${ }^{30}$ The use of different tools makes comparison difficult across studies. ${ }^{20,26}$ However, despite these methodological and contextual differences, there are aspects that are worthy of further exploration, such as gender 
differences. With a generally gendered workforce, it is important to further understand areas relating to care provision where males and females may differ as such differences can be a threat to consistency and model of care in practice. One hypothesis may be that female staff see relational aspects of care as more naturally occurring and thus empirically present compared to males, or alternatively that male staff can be focused on procedural aspects of care and interventions. Such differences may need further exploration and reflection in the literature. Age differences may be another such aspect in need of further study. It seems reasonable to hypothesize that with age comes increased experiences, and that such experiences facilitate the movement from novice to expert as described in Benner and Wrubel's seminal work ${ }^{31}$ including relational and person-centered aspects.

The study findings can be interpreted as having practical implications for assessing and improving the psychosocial climate of the organization and work conditions, through reporting covariates and content of person-centered care that can be further explored in practice development initiatives. Also, measuring the degree of person-centeredness can and should be connected in the future to other measurements of care, as there are emerging links between PCC and patient outcomes, staff satisfaction, job stress, communication, and work burnout. ${ }^{20,32}$ Perceptions of individuals are very important to clarify, as a baseline for setting the tone and direction for person-centered care. ${ }^{33,34}$

\section{Limitations}

Due to the cross-sectional design of the study, the generalizability of the findings is limited. Moreover, this study is concerned with measuring the psychosocial climate using a self-reporting process; its validity may be threatened by common method variance, which can lead to positive or negative correlation among variables. In other words, a true measurement of the psychosocial climate cannot be achieved because the accuracy of measurement will always be limited to some degree by the method we have used, including the social desirability bias that might tend to happen when respondents feel the matter is sensitive, so they do not want to reveal their true feelings about what is actually happening. Thus, the mixed methods approach would be useful to further assess the aspects and covariates of PCC as highlighted in this study, for example, relating to age and gender; however, due to resource constraints in this study, a strict quantitative approach was used without possibilities for further exploration narratively. The study sample reflects the views of HCPs from different disciplines. However, the sample lacks the views of patients groups due to the staff population. The limited explanatory capacity of the model is also a weakness and a call for further studies, as there seems to be a large proportion of unexplained variance that influences PCC perceptions among HCPs, and further understanding the additional explanatory variables for PCC is essential to further build this knowledge base and practice development readiness.

\section{Conclusions}

This study investigated health care providers' perspectives on person-centered care and to identify if health care providers' characteristics were related to the perception of a personcentered care climate. Based on our findings, there is a room for PCC climate improvement since the person-centeredness climate scores were comparatively low in light of previously reported scores in the literature. The study findings indicated that the main factors associated with HCPs' perception of PCC were higher age and female gender, and these factors would benefit from further exploration in research and practice to further understand and implement standards for PCC. It has been shown that person-centered care impacts the quality of care provided by an organization, and these study findings can be used for quality improvement projects designed primarily to improve the PCC climate in a hospital care setting, at least partly by building practice development conversations on the items of the PCCQ tool as an evidence base. Although the overall PCC climate needs more attention from the health care leadership, the interventions priority should be given to everydayness followed by community and safety.

\section{Abbreviations}

HCPs, Health Care Providers; IOM, Institute of Medicine; PCC, Person-Centered Care; PCQ-S, Person-Centered Climate Questionnaire-Staff Version; SE, Standard Error; SD, Standard Deviation; SAS, Statistical Analysis Software.

\section{Data Sharing Statement}

The datasets generated and/or analyzed during the current study are not publicly available due to the institutional rules and regulations but are available from the first author on a reasonable request.

\section{Consent for Publication}

The participants were informed of the ethical approval of the project. 


\section{Author Contributions}

All authors made substantial contributions to conception and design, acquisition of data, or analysis and interpretation of data; took part in drafting the article or revising it critically for important intellectual content; agreed to submit to the current journal; gave final approval for the version to be published; and agreed to be accountable for all aspects of the work.

\section{Funding}

The authors received no specific funding for this research.

\section{Disclosure}

The authors declare no conflict of interest.

\section{References}

1. Luxford K, Safran DG, Delbanco T. Promoting patient-centered care: a qualitative study of facilitators and barriers in healthcare organizations with a reputation for improving the patient experience. Int J Qual Health Care. 2011;23(5):510-515. doi:10.1093/intqhe/ mzr024

2. Rathert C, Wyrwich MD, Boren SA. Patient-centered care and outcomes: a systematic review of the literature. Med Care Res Rev. 2013;70(4):351-379. doi:10.1177/1077558712465774

3. Frampton SB, Guastello S, Lepore M. Compassion as the foundation of patient-centered care: the importance of compassion in action. J Comp Eff Res. 2013;2(5):443-455. doi:10.2217/cer.13.54

4. Weingart SN, Page D. Implications for practice: challenges for healthcare leaders in fostering patient safety. BMJ Qual Saf. 2004;13(suppl 2):ii52-ii56.

5. Rocco N, Scher K, Basberg B, Yalamanchi S, Baker-Genaw K. Patient-centered plan-of-care tool for improving clinical outcomes. Qual Manag Health Care. 2011;20(2):89-97. doi:10.1097/ QMH.0b013e318213e728

6. Hudon C, Fortin M, Haggerty JL, Lambert M, Poitras M-E. Measuring patients' perceptions of patient-centered care: a systematic review of tools for family medicine. Ann Fam Med. 2011;9(2):155-164. doi:10.1370/afm.1226

7. Pelletier LR, Stichler JF. Patient-centered care and engagement: nurse leaders' imperative for health reform. $J$ Nurs Adm. 2014;44 (9):473-480. doi:10.1097/NNA.0000000000000102

8. Choi J, Kim DE, Yoon JY. Person-centered care environment associated with care staff outcomes in long-term care facilities. $J$ Nurs Res. 2020;29(1):e133. doi:10.1097/JNR.0000000000000412

9. Sidani S, Fox M. Patient-centered care: clarification of its specific elements to facilitate interprofessional care. J Interprof Care. 2014;28 (2):134-141. doi:10.3109/13561820.2013.862519

10. Kirkley C, Bamford C, Poole M, Arksey H, Hughes J, Bond J. The impact of organisational culture on the delivery of person-centred care in services providing respite care and short breaks for people with dementia. Health Soc Care Community. 2011;19(4):438-448.

11. World Health Organization. WHO Global Strategy on PeopleCentered and Integrated Health Services: Interim Report; 2015.

12. Department of Health \& Human Services. What is Person-Centred Health Care? A Literature Review; 2011.

13. Robinson JH, Callister LC, Berry JA, Dearing KA. Patient-centered care and adherence: definitions and applications to improve outcomes. J Am Acad Nurse Pract. 2008;20(12):600-607. doi:10.1111/ j.1745-7599.2008.00360.x
14. Hobbs JL. A dimensional analysis of patient-centered care. Nurs Res. 2009;58(1):52-62. doi:10.1097/NNR.0b013e31818c3e 79

15. Lusk JM, Fater K. A concept analysis of patient-centered care. In: Nursing Forum. Vol. 48. Wiley Online Library; 2013:89-98.

16. Ebrahimi Z, Patel H, Wijk H, Ekman I, Olaya-Contreras P. A systematic review on implementation of person-centered care interventions for older people in out-of-hospital settings. Geriatr Nurs. 2021;42(1):213-224. doi:10.1016/j.gerinurse.2020.08.004

17. Edvardsson D, Sjögren K, Lindkvist M, Taylor M, Edvardsson K, Sandman PO. Person-centred climate questionnaire (PCQ-S): establishing reliability and cut-off scores in residential aged care. $J$ Nurs Manag. 2015;23(3):315-323. doi:10.1111/jonm.12132

18. Schneider B, Hanges PJ, Smith DB, Salvaggio AN. Which comes first: employee attitudes or organizational financial and market performance? J Appl Psychol. 2003;88(5):836. doi:10.1037/00219010.88.5.836

19. Park M, Jeong H, Giap -T-T-T. A predictive model of the perceptions of patient-centered care among nurses in long-term care hospitals: a cross-sectional study. Geriatr Nurs. 2021;42(3):687-693. doi:10.1016/j.gerinurse.2021.02.019

20. Sjogren K, Lindkvist M, Sandman P-O, Zingmark K, Edvardsson D. To what extent is the work environment of staff related to person-centred care? A cross-sectional study of residential aged care. J Clin Nurs. 2015;24(9-10):1310-1319. doi:10.1111/jocn.12734

21. Edvardsson D, Koch S, Nay R. Psychometric evaluation of the English language person-centred climate questionnaire-staff version. $J$ Nurs Manag. 2010;18(1):54-60. doi:10.1111/j.1365-2834.2009.01038.x

22. Lehuluante A, Nilsson A, Edvardsson D. The influence of a personcentred psychosocial unit climate on satisfaction with care and work. $J \quad$ Nurs Manag. 2012;20(3):319-325. doi:10.1111/j.13652834.2011.01286.x

23. Wilde-Larsson B, Inde M, Carlson A, Nordström G, Larsson G, Rystedt I. Implementation of patient-focused care: before-after effects. Int $J$ Health Care Qual Assur. 2014;27(7):594-604. doi:10.1108/IJHCQA-06-2013-0067

24. Koch L. Individualized Care and Rehabilitation; 2019:151-162.

25. Sawamura K, Nakashima T, Nakanishi M. Provision of individualized care and built environment of nursing homes in Japan. Arch Gerontol Geriatr. 2013;56(3):416-424. doi:10.1016/j.archger.2012.11.009

26. Berghout M, van Exel J, Leensvaart L, Cramm JM. Healthcare professionals' views on patient-centered care in hospitals. $B M C$ Health Serv Res. 2015;15(1):385. doi:10.1186/s12913-015-1049-z

27. Leplege A, Gzil F, Cammelli M, Lefeve C, Pachoud B, Ville I. Personcentredness: conceptual and historical perspectives. Disabil Rehabil. 2007;29(20-21):1555-1565. doi:10.1080/09638280701618661

28. Jorm CM, Dunbar N, Sudano L, Travaglia JF. Should patient safety be more patient centred? Aust Health Rev. 2009;33(3):390-399. doi:10.1071/AH090390

29. Harris PB, McBride G, Ross C, Curtis L. A place to heal: environmental sources of satisfaction among hospital patients. J Appl Soc Psychol. 2002;32(6):1276-1299. doi:10.1111/j.1559-1816.2002.tb01436.x

30. Yang Y, Li H, Xiao LD, Zhang W, Xia M, Feng H. Resident and staff perspectives of person-centered climate in nursing homes: a cross-sectional study. BMC Geriatr. 2019;19(1):292. doi:10.1186/ s12877-019-1313-x

31. Edwards SD. Benner and Wrubel on caring in nursing. $J$ Adv Nurs. 2001;33(2):167-171. doi:10.1046/j.1365-2648.2001.01649.x

32. Stewart M, Brown JB, Donner A, et al. The impact of patient-centered care on outcomes. J Fam Pract. 2000;49(9):796-804.

33. Johnson BH, Abraham MR, Shelton TL. Patient- and family-centered care: partnerships for quality and safety. $N$ C Med J. 2009;70 (2):125-130.

34. Ivany A, LeBlanc C, Grisdale M, Maxwell B, Langley JM. Reducing infection transmission in the playroom: balancing patient safety and family-centered care. Am J Infect Control. 2016;44(1):61-65. doi:10.1016/j.ajic.2015.07.036 


\section{Publish your work in this journal}

Risk Management and Healthcare Policy is an international, peerreviewed, open access journal focusing on all aspects of public health, policy, and preventative measures to promote good health and improve morbidity and mortality in the population. The journal welcomes submitted papers covering original research, basic science, clinical \& epidemiological studies, reviews and evaluations, guidelines, expert opinion and commentary, case reports and extended reports. The manuscript management system is completely online and includes a very quick and fair peer-review system, which is all easy to use. Visit http://www.dovepress.com/testimonials.php to read real quotes from published authors.

Submit your manuscript here: https://www.dovepress.com/risk-management-and-healthcare-policy-journal 